\title{
Normalized Partial Scattering Cross Section for Performance Evaluation of Low-Observability Scattering Structures
}

\author{
Muhammad Abdullah ${ }^{1}$, Slawomir Koziel ${ }^{1,2, *(D)}$ and Stanislaw Szczepanski ${ }^{2}$ \\ 1 Engineering Optimization and Modeling Center, Reykjavik University, 101 Reykjavik, Iceland; \\ muhammada19@ru.is \\ 2 Faculty of Electronics, Telecommunications and Informatics, Gdańsk University of Technology, \\ 80-233 Gdańsk, Poland; stanislaw.szczepanski@pg.edu.pl \\ * Correspondence: koziel@ru.is
}

\section{check for} updates

Citation: Abdullah, M.; Koziel, S.; Szczepanski, S. Normalized Partial Scattering Cross Section for Performance Evaluation of Low-Observability Scattering Structures. Electronics 2021, 10, 1731 https://doi.org/10.3390/ electronics10141731

Academic Editors: Luciano De

Tommasi, Ivo Couckuyt and

Domenico Spina

Received: 23 June 2021

Accepted: 16 July 2021

Published: 19 July 2021

Publisher's Note: MDPI stays neutral with regard to jurisdictional claims in published maps and institutional affiliations.

Copyright: (c) 2021 by the authors. Licensee MDPI, Basel, Switzerland. This article is an open access article distributed under the terms and conditions of the Creative Commons Attribution (CC BY) license (https:// creativecommons.org/licenses/by/ $4.0 /)$.

\begin{abstract}
The development of diffusion metasurfaces created new opportunities to elevate the stealthiness of combat aircraft. Despite the potential significance of metasurfaces, their rigorous design methodologies are still lacking, especially in the context of meticulous control over the scattering of electromagnetic (EM) waves through geometry parameter tuning. Another practical issue is insufficiency of the existing performance metrics, specifically, monostatic and bistatic evaluation of the reflectivity, especially at the design stage of metasurfaces. Both provide limited insight into the RCS reduction properties, with the latter being dependent on the selection of the planes over which the evaluation takes place. This paper introduces a novel performance metric for evaluating scattering characteristics of a metasurface, referred to as Normalized Partial Scattering Cross Section (NPSCS). The metric involves integration of the scattered energy over a specific solid angle, which allows for a comprehensive assessment of the structure performance in a format largely independent of the particular arrangement of the scattering lobes. We demonstrate the utility of the introduced metric using two specific metasurface architectures. In particular, we show that the integral-based metric can be used to discriminate between the various surface configurations (e.g., checkerboard versus random), which cannot be conclusively compared using traditional methods. Consequently, the proposed approach can be a useful tool in benchmarking radar cross section reduction performance of metamaterial-based, and other types of scattering structures.
\end{abstract}

Keywords: diffusion; metasurfaces; radar cross section (RCS); performance metrics; bistatic scattering

\section{Introduction}

In recent years, metasurfaces, with their unique ability to shape the electromagnetic (EM) wavefront, have attracted increasing attention from researchers and practitioners [1-3]. Some of the areas where metasurfaces found their applications include radar cross section (RCS) reduction, as well as spatial processing, and polarization conversion techniques [4-6]. The advancements of radar detection technology placed RCS reduction in a forefront of research on stealth technology [7]. To date, metasurfaces have been extensively exploited to accomplish essential RCS reduction of the targets $[8,9]$. One practical design strategy in this regard is to utilize metamaterials, particularly, exploiting their distinctive property of manipulating the phase reflection characteristics [10]. It entails a periodic combination of artificial magnetic conductors (AMC), and perfect electric conductors (PEC), arranged to attain the desired phase reflection characteristics. To extend the RCS reduction bandwidth of such structures, the concepts of coding metasurfaces [11], diffusion metasurfaces [12,13], programmable metasurfaces [14], Huygens' metasurfaces [15], as well as cloaking structures [16], have been proposed, which offers control over the wavefront in a more sophisticated manner. The primary advantage of coding and diffusion metasurfaces is that it scatters the incident EM waves into all directions. In addition to that, coding metasurfaces are also being exploited as an absorptive surface to realize essential RCS reduction [17]. 
The design of metasurfaces requires availability of performance metrics that can be applied for their assessment, as well as to discriminate between alternative architectures. The existing metrics developed to measure RCS reduction performance of a diffusion metasurface are based on evaluating the energy scattered from the target with reference to the PEC surface featuring the same physical dimension. The scattering characteristics can be studied in monostatic and bistatic modes of operation [18-23]. The former conforms to a situation where the transmitter and the receiver radars are collocated, and, hence, RCS performance of an aircraft is evaluated with respect to a specified position [24]. This approach provides limited insight into the metasurface properties. In a related vein, a bistatic mode corresponds to a condition when the transmitter and receiver radars are stationed at a certain distance between them [24]. Unfortunately, bistatic RCS performance is highly dependent on the selection of the planes over which the evaluation takes place [25], which makes it inconvenient as an evaluation tool for RCS-reduction-oriented metasurface design. In Reference [26], a figure of merit for bistatic RCS reduction (FMB) has been introduced (based on the maximum RCS), which is a step forward in making RCS evaluation angle-independent. However, this metric boils down to considering the worst-case scenario performance, which may provide an overly pessimistic evaluation because it is related to the maximum reflection lobe of a small angular spread. With the development of multi-base radar detection technology, the accomplishment of bistatic RCS reduction turns out to be a measure of choice, together with the monostatic RCS. Notwithstanding, comprehensive quantification of the scattered energy in a regime that is independent of an arbitrary selection of the specific setup, requires the development of more sophisticated measures of merit. These should be pertinent to both contemporary needs (as the aforementioned multi-base radars), and the various types of metasurfaces featuring distinct scattering patterns.

This paper introduces a novel performance metric for evaluating scattering properties of metasurfaces, which is intended to deliver an effective tool for comprehensive assessment of RCS reduction characteristics. The metric, referred to as Normalized Partial Scattering Cross Section (NPSCR), involves numerical integration of the energy scattered from the target over a solid angle. As a result, a conclusive assessment concerning observability properties of the diffusion metasurface can be obtained in a way independent of the particulars of the setup. Furthermore, the metric evaluates the average performance, which is not biased towards a particular scattering pattern. We demonstrate the significance of the proposed metric through benchmarking of two specific metasurface architectures. In particular, we show that the integral-based metric allows for meaningful discrimination between alternative metasurface setups (e.g., checkerboard versus random) even though traditional metrics remain inconclusive. The results indicate that the proposed metric may be a useful tool in the design of scattering metasurfaces featuring improved RCSreduction performance.

The remaining part of the article is organized as follows. Section 2 discusses the motivation of the introduced Normalized Partial Scattering Cross Section (NPSCR). In Section 3, the underlying concepts behind RCS of scattering metasurfaces are provided, followed by a brief description of the proposed integral-based metric. In Section 4, we demonstrate the significance and practical utility of the integral-based metric using two benchmark examples. Section 5 concludes this paper.

\section{Motivation}

The key performance indicator in the design of low scattering metasurface is the RCS reduction characteristics. The design can be evaluated under two types of scattering conditions, i.e., the monostatic or bistatic ones. Therein, a realistic environment can be characterized to assess the practical performance of a surface.

The advent of a multi-base radar detection technology demands contemporary stealth structures to feature nominal RCS. The standard monostatic and bistatic regimes are illustrated in Figure 1. As shown, the transmitter and the receiver radars are separated by a 
certain distance. In a bistatic mode, the angle between the receiver radar and reflected EM waves are denoted by $\beta$ and is referred to as a bistatic angle [27]. It can be observed from Figure 1 that the bistatic RCS solely depends on the selection of the measuring plane, i.e., it is increased whenever the plane misses the scattering lobes and drops otherwise. Therefore, the nature of bistatic characteristics restricts their evaluation to only a single direction (angle) at a specific instance. Consider Figure 2, where the bistatic RCS performance of a considered metasuface is evaluated along the three scattering angles, $\beta=10^{\circ}, 35^{\circ}$, and $75^{\circ}$. Expectedly, the results are dependent on the angle of observation. In particular, the best RCS reduction does not endure at all of the tested angles, and similarly, it varies along the plane [27-29].

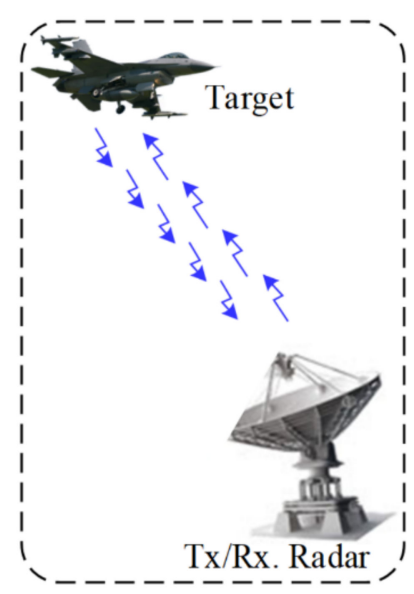

(a)

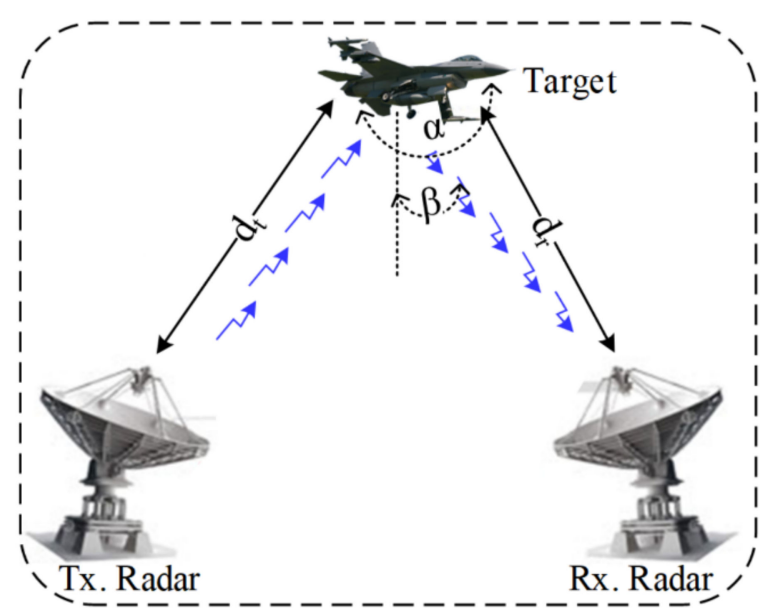

(b)

Figure 1. Illustration of (a) a monotonic, and (b) bistatic mode of operation.

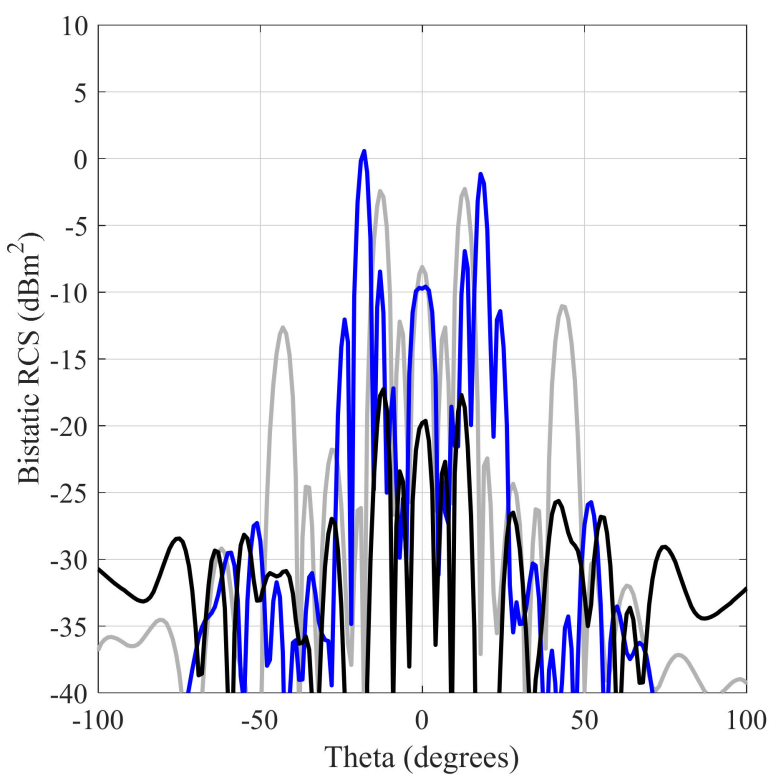

Figure 2. Exemplary bistatic RCS characteristics at an observation angle $\beta=10^{\circ}$ (gray), $35^{\circ}$ (blue) and $75^{\circ}$ (black).

This implies that bistatic performance evaluated at a few selected angles or planes is insufficient to deliver a comprehensive account for the metasurface properties, which becomes problematic especially in the design process of high-performance RCS-reduction metasurfaces. The bistatic RCS characteristics given in Figure 2 are obtained by evaluating metasurface IV (cf. Section 4). Consequently, a new metric should be developed that 
alleviates the difficulties of the existing methods and is more aligned with the contemporary needs, including those pertinent to design procedures. In this regard, evaluating RCS properties along a specified angular spread (e.g., a cone determined by a solid angle $\alpha$, see Figure 1) may deliver a more conclusive assessment of the bistatic performance of a surface.

\section{Proposed Metric}

This section briefly describes the underlying concepts behind RCS of scattering metasurfaces, and anchor these to monostatic and bistatic characteristics. Later on, we give a formal definition of a novel performance metric proposed in this work. Its utility in the context of metasurface design and evaluation will be demonstrated in Section 4.

\subsection{Background Information}

The mechanism of a monostatic and bistatic RCS can be understood by recalling a planar array excited with a progressive phase shift of $180^{\circ}$ over a certain frequency band $[23,300]$. The metasurface exploits the anti-phase reflection property of periodic arrays to control the scattered energy.

To quantitatively illustrate the physical phenomenon, we consider a generalized configuration of a metasurface (cf. Figure 3 ) that contains $N \times N$ equally-sized lattices of dimension $d$. The scattering phase of each lattice is assumed to be $\phi(m, n)$. Under the normal incidence of the plane waves, the far-field array factor corresponding to the energy scattered by a metasurface [23] is given as

$$
\begin{gathered}
\operatorname{AF}(\theta, \phi, f)=E_{e}(\theta, \phi, f) \\
\sum_{m=1}^{N} \sum_{n=1}^{N} \exp \{-i\{\phi(m, n)+k d \sin \theta[(m-1 / 2) \cos \phi+(n-1 / 2) \sin \phi]\}\}
\end{gathered}
$$

where $\theta$ and $\varphi$ denote the elevation and the azimuth angles of a scattering direction, respectively, $f$ denotes the specified frequency and $E_{e}(\theta, \phi, f)$ is the pattern function of a lattice at a frequency $f$. Subsequently, the overall directivity of the metasurface can be obtained as

$$
\mathrm{D}(\theta, \phi, f)=\frac{4 \pi|\mathrm{AF}(\theta, \phi, f)|^{2}}{\int_{0}^{180} \int_{0}^{90}|\operatorname{AF}(\theta, \phi, f)|_{2} \sin \theta \mathrm{d} \theta \mathrm{d} \phi}
$$

On the other hand, the principle of RCS reduction is based upon redirecting the incident EM energy to all directions in the space. More specifically, the RCS of a corresponding surface at the scattering angles $\theta$ and $\phi$ at the frequency $f$ can be expressed as

$$
\sigma(\theta, \phi, f)=\lambda^{2}\left[4 \pi \mathrm{N}^{2} d^{2}\right]^{-1} \mathrm{D}(\theta, \phi, f)
$$

where $\lambda$ is the free space wavelength.

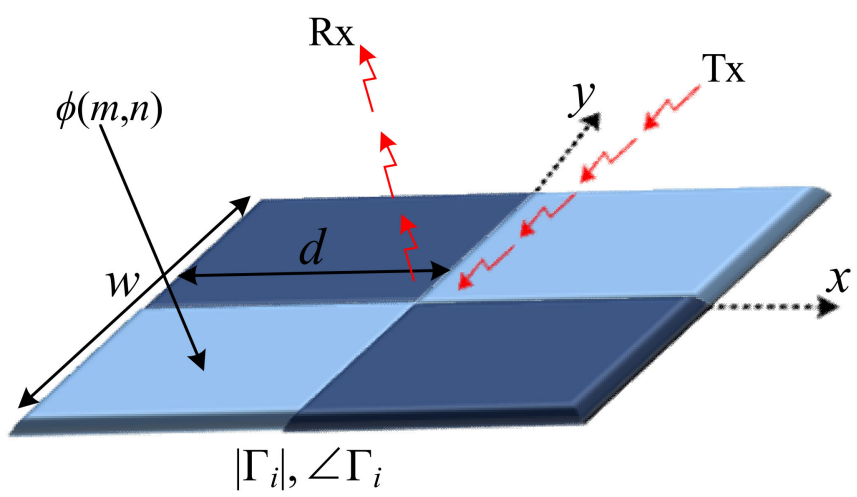

Figure 3. A simple configuration of a $N \times N$ metasurface. 
Note that the monostatic RCS refers to a specific $\theta$ and $\varphi$ (e.g., $\sigma(0,0, f)$, where $\theta=0^{\circ}$ and $\left.\varphi=0^{\circ}\right)$ at a frequency $f$. Alternatively, bistatic RCS corresponds to $\sigma(\theta, \varphi, f)$, with $-90^{\circ} \leq \theta \leq 90^{\circ}$, whereas $\varphi$ determines the evaluation plane (e.g., $0^{\circ}$ or $90^{\circ}$ ).

\subsection{Novel Integral-Based Performance Metric}

As indicated in Section 1, characterization of the RCS reduction using both the monostatic and bistatic approaches is generally insufficient in terms of providing comprehensive assessment of metasurface performance, especially in the light of modern circumstances (e.g., multi-base radars). Furthermore, bistatic RCS may be misleading due to being highly dependent on the choice of the evaluation planes.

In order to mitigate these issues, this work proposes a novel, integral-based, performance metric, referred to as Normalized Partial Scattering Cross Section (NPSCR). It is be defined as the metric involving numerical integration of the energy scattered from the target over a solid angle $\Omega=(\theta, \phi) \in\left[\theta_{\min }, 90^{\circ}\right] \times\left[-180^{\circ}, 180^{\circ}\right]$, where $\theta_{\min }$ is the angle defining the considered range $\Omega$. Analytically, it is formulated as

$$
\sigma_{\Omega}(f)=\left|\frac{\oiint \sigma(\Omega, f) d \Omega}{\oiint \sigma^{P}(\Omega, f) d \Omega}\right|
$$

where $\sigma^{P}$ is the RCS of a perfect electric conductor (PEC) having the same physical dimension. In other words, $\sigma_{\Omega}$ represents the RCS of the corresponding metasurface averaged over a solid angle $\Omega$ normalized to the same of the PEC surface. A scalarized version of the metric, which may be more convenient for, e.g., design optimization purposes, is defined as

$$
\sigma=\left[f_{U}-f_{L}\right]^{-1} \int_{f_{L}}^{f_{U}} \sigma_{\Omega}(f) d f
$$

where $f_{L}$ and $f_{U}$ determine the frequency range of interest. Thus, $\sigma$ is the average RCS performance over the frequency interval $\left[f_{L}, f_{H}\right]$.

An important advantage of the metrics (4) and (5) is that it is not biased towards a particular scattering pattern. As a result, a conclusive assessment concerning the observable properties of the diffusion metasurface can be obtained in a way independent of the particulars of the setup (e.g., the choice of the evaluation plane for a bistatic mode). It should be noted that NPSCR exhibits certain resemblance to the Scattering Cross Section [30], although it is more specific in terms of being defined over a pre-defined frequency spectrum and the angular spread of the scattering directions.

\section{Numerical Results and Discussions}

This section demonstrates the significance and practical utility of the integral-based metric introduced in Section 3, using two specific metasurface examples. We start by benchmarking the two architectures of similar underlying designs but with different arrangement of the lattices. Subsequently, we consider performance discrimination between the four alternative metasurface designs.

\subsection{Case Study I}

Our first verification case is a coding metasurface employing crusader-cross-shaped unit cells. The structure is implemented on a ground-backed Arlon AD250 lossy substrate $(\varepsilon r=2.5$, $h=1.5 \mathrm{~mm}, \tan \delta=0.0018$ ). The overall size of each surface is $W \times L=144 \times 144 \mathrm{~mm}^{2}$. The topology of the cell, and the two configurations of the metasurface are shown in Figures 4 and 5, respectively. For the sake of brevity, we omit the detailed information about cell parameterization (cf. [31]). The CST Microwave Studio is used to evaluate the field performance of all metasurfaces considered in this work. 


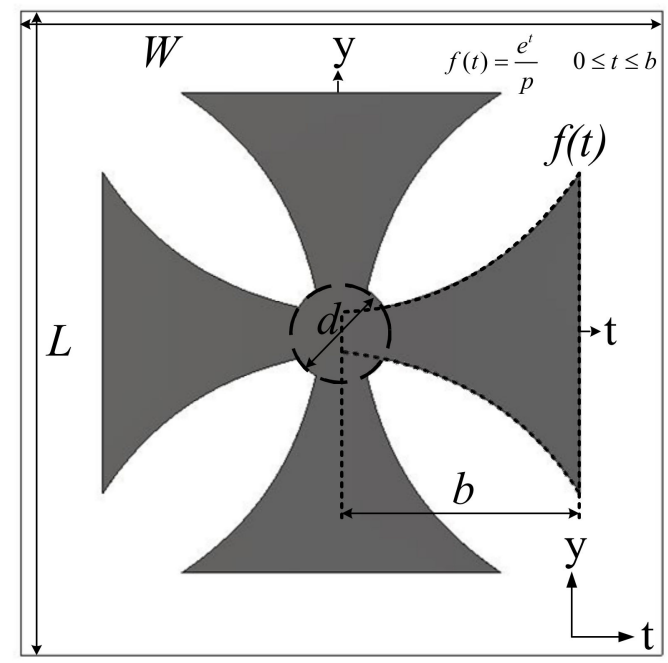

Figure 4. Topology of the crusader-cross-shaped unit cell.

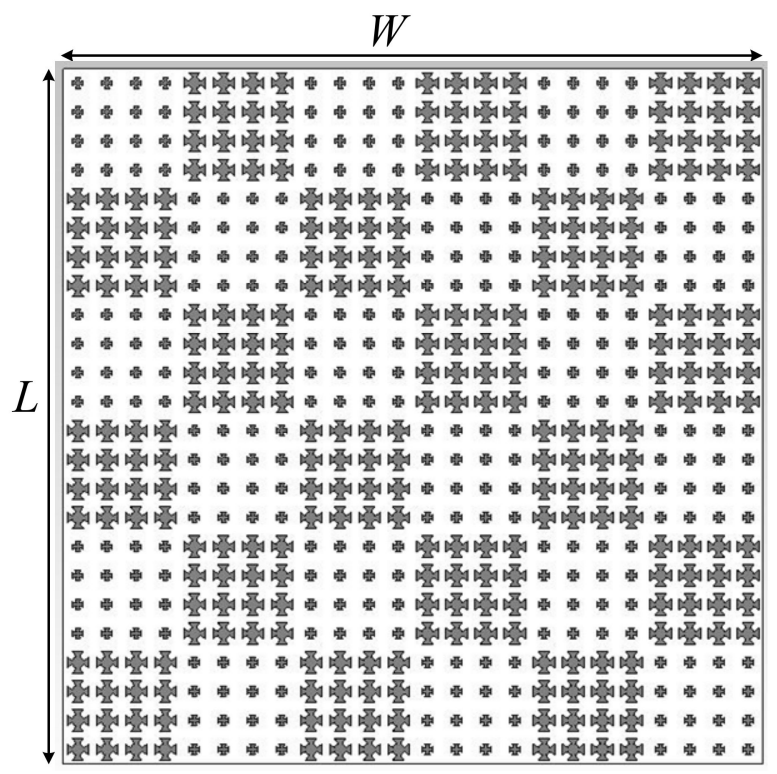

(a)

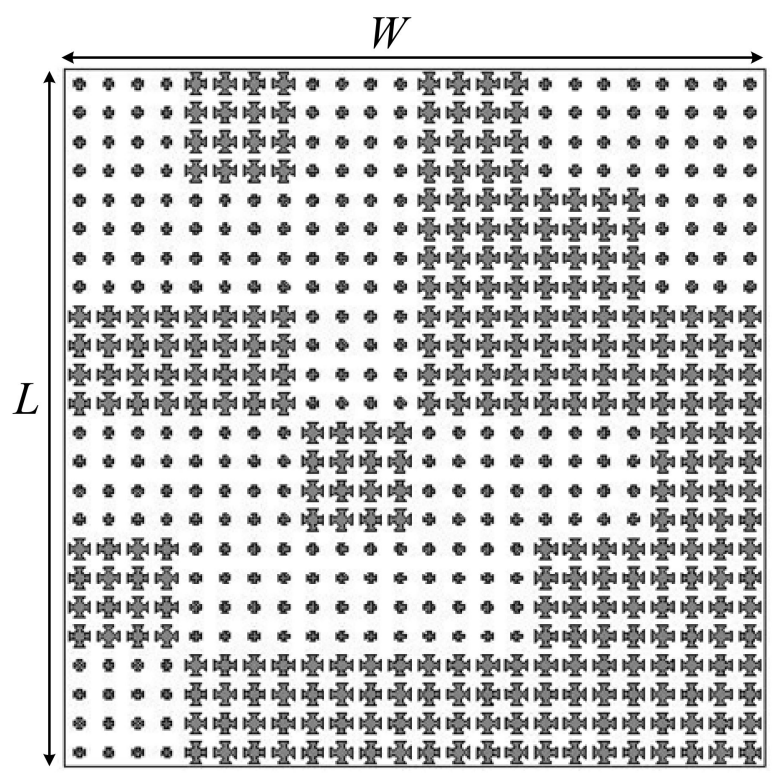

(b)

Figure 5. (a) Configuration of the checkerboard; (b) optimized metasurface architectures.

One of the considered metasurfaces is a standard checkerboard configuration, whereas in the second case, the composition of the lattices is determined by globally optimizing (1) using binary coded genetic algorithm, as presented in [28].

Our target is to assess the RCS reduction performance of the two architectures, thus determining the recommended structure. First, we attempt to discriminate between the two structures by means of the existing measures of merits, i.e., the monostatic and bistatic RCS. Figure 6 shows the monostatic and bistatic RCS performance of the two designs. It can be observed that neither of these metrics allows for conclusive determination of the betterperforming structure. On the one hand, considering the monostatic RCS characteristics, the optimized metasurface is superior. On the other hand, the checkerboard architecture features lower bistatic RCS than the optimized surface; however, this is only for a specific plane, and a specific frequency. As mentioned before, bistatic evaluation is ambiguous mainly due to dependence of the plane selection and the particular scattering pattern of the surface. 

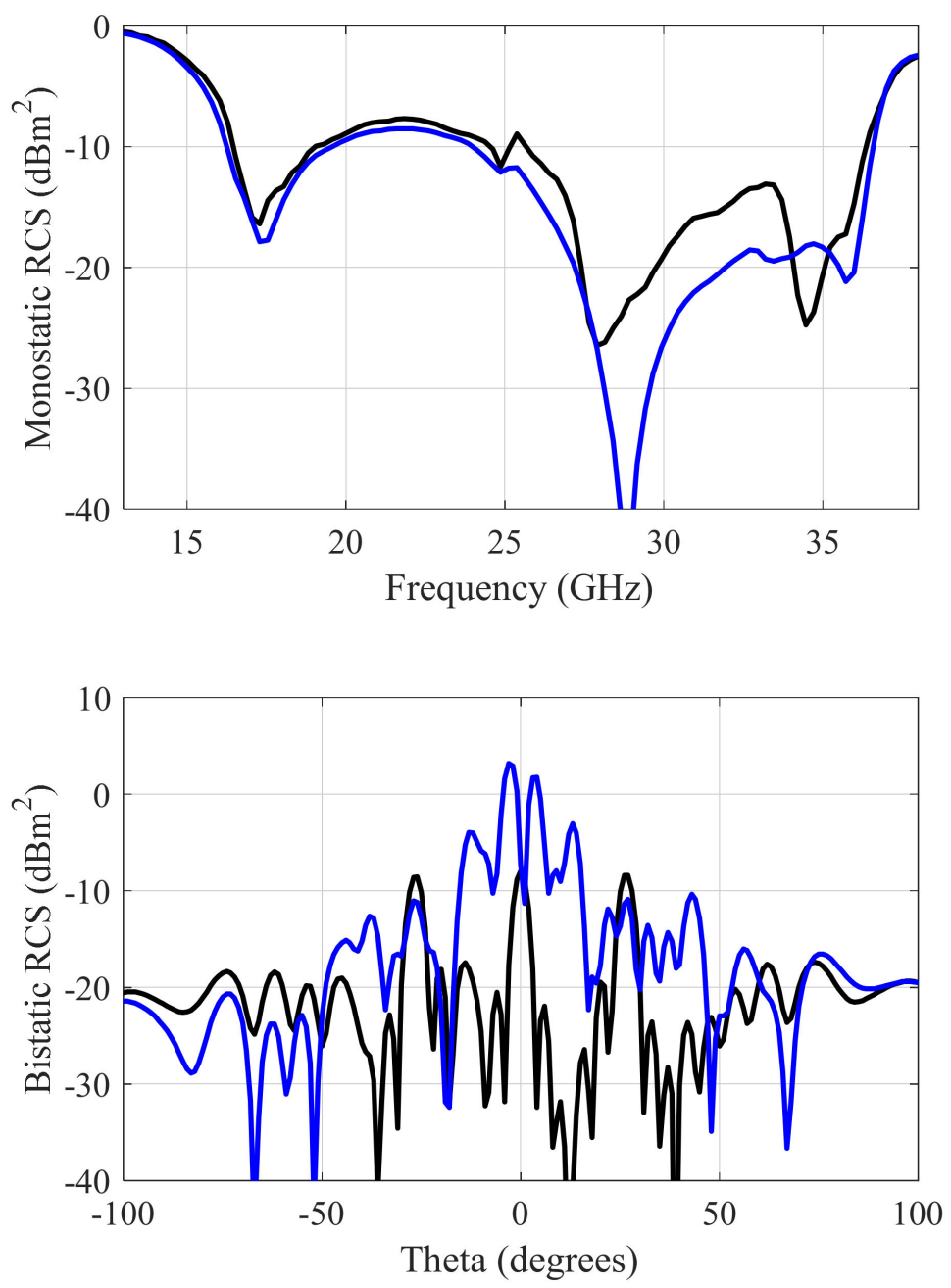

Figure 6. Monostatic (top) and bistatic at $28 \mathrm{GHz}$ (bottom) RCS characteristics of the two metasurfaces. The bistatic RCS is evaluated at $\theta=0^{\circ}$ and $\varphi=0^{\circ}$. The monostatic performance is normalized to the reference PEC surface. The black and blue color corresponds to checkerboard and optimized metasurface performance, respectively.

At this point, we apply the proposed integral-based metric to evaluate the considered architectures. Figure 7 shows two cases, parameterized by the solid angle: $\theta_{\min }=30^{\circ}$ (i.e., $\Omega=\left[30^{\circ}, 90^{\circ}\right] \times\left[-180^{\circ}, 180^{\circ}\right]$ ) and $\theta_{\min }=45^{\circ}$ (i.e., $\Omega=\left[45^{\circ}, 90^{\circ}\right] \times\left[-180^{\circ}, 180^{\circ}\right]$ ) (cf. Section 3.2). It can be observed that regardless of $\theta_{\min }$, the optimized metasurface features better RCS reduction properties than the checkerboard version over a wide range of frequencies. Furthermore, the average RCS performance (cf. (5)) for the checkerboard and the optimized metasurfaces is 11.49 and 20.87 for $\theta_{\min }=30^{\circ}$; for $\theta_{\min }=45^{\circ}$ the figures are 11.56 and 20.89, respectively. Please note that the end-result in Figure 7 involves integration of the overall scattering performance of the metasurface, therefore, the response at two solid angles, i.e., at $\theta_{\min }=30^{\circ}$ and $\theta_{\min }=45^{\circ}$, seem to be almost the same. Thus, the optimized metasurface is conclusively superior in terms of the integral-based metric. 

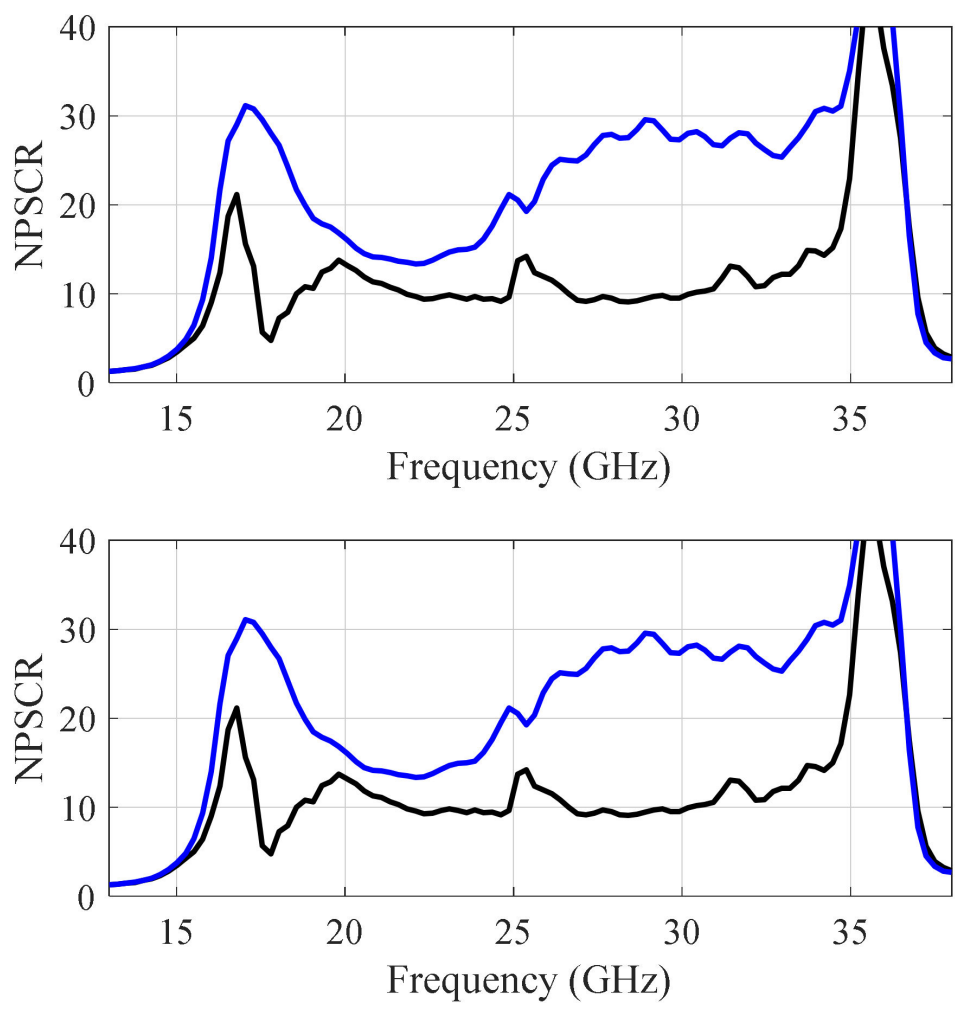

Figure 7. RCS performance evaluated using NPSCS of (4) when $\theta_{\min }=30^{\circ}$ (top) and when $\theta_{\min }=45^{\circ}$ (bottom). The black and blue color corresponds to checkerboard and optimized metasurface performance, respectively.

\subsection{Case Study II}

The second demonstration example involves four metasurfaces implemented using entirely different unit cell topologies as shown in Figure 8. The first metasurface is based on a design of [25], the second, and the third ones were presented in [28,29], respectively, hereafter referred to as metasurface I, II, and III, respectively. The fourth surface involves the underlying unit cell topology as given in Figure 4, hereafter referred to as metasurface IV. All the designs are further adjusted to obtain the optimal performance in the sense of maximizing monostatic RCS reduction bandwidth.

In this study, the aim is to quantify the RCS reduction performance of these metasurface architectures using the integral-based metric. For fair assessment, the comparison is carried out in terms of the average RCS performance (5) calculated over the frequency intervals corresponding to the $10 \mathrm{~dB}$ monostatic RCS reduction bandwidths of the respective surfaces. For each case, five angular spreads are considered, parameterized by the solid angle: $\theta_{\min }=10^{\circ}, 20^{\circ}, 30^{\circ}, 40^{\circ}, 50^{\circ}$ (i.e., $\Omega=\left[\theta_{\min }, 90^{\circ}\right] \times\left[-180^{\circ}, 180^{\circ}\right]$ ), cf. Section 3.2. The results are presented in Table 1 . It can be observed that metasurfaces III and IV are superior over metasurfaces I and II for $\theta_{\min } \geq 20^{\circ}$, and their performance is similar to each other. This means that for the vast majority of operating conditions in terms of $\theta_{\min }$, the last two structures are conclusively better and the difference is significant (performance improvement by a factor of about 2.5 over the first two structures). Only for the lowest value, $\theta_{\min }=10^{\circ}$, does metasurface I exhibit the best properties. 


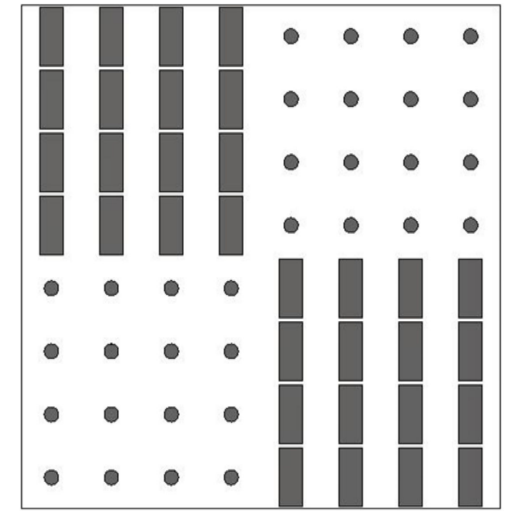

(a)

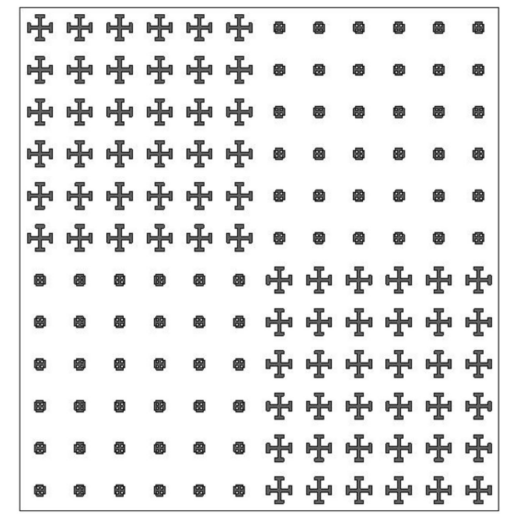

(c)

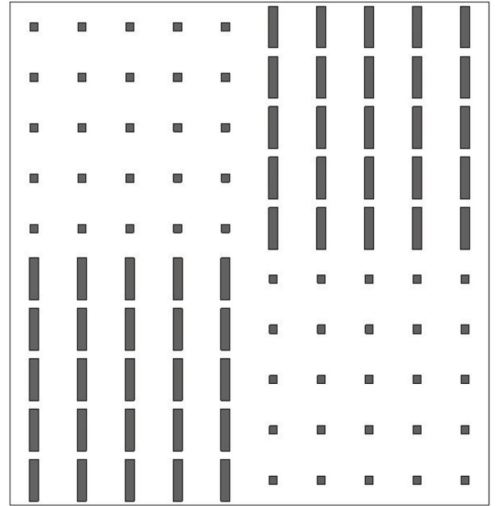

(b)

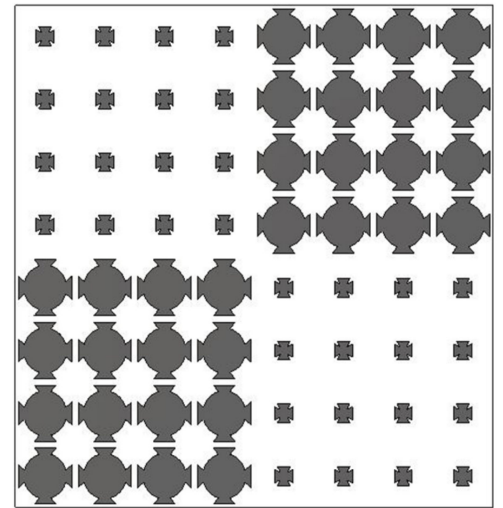

(d)

Figure 8. Configuration of the metasurfaces; (a) metasurface I [25], (b) metasurface II [28], (c) metasurface III [29], and (d) metasurface IV.

Table 1. Performance Comparison of Different Metasurface Designs.

\begin{tabular}{ccccccc}
\hline \multirow{2}{*}{ Design } & \multirow{2}{*}{$\begin{array}{c}\text { Polarization } \\
\text { Dependent }\end{array}$} & \multicolumn{5}{c}{ Average RCS Performance (5) } \\
\cline { 3 - 7 } & $\boldsymbol{\theta}_{\text {min }}=\mathbf{1 0}^{\circ}$ & $\boldsymbol{\theta}_{\text {min }}=\mathbf{2 0}^{\circ}$ & $\boldsymbol{\theta}_{\text {min }}=\mathbf{3 0}^{\circ}$ & $\boldsymbol{\theta}_{\text {min }}=\mathbf{4 0}^{\circ}$ & $\boldsymbol{\theta}_{\text {min }}=\mathbf{5 0}^{\circ}$ \\
\hline Metasurface I & Yes & 37.0 & 5.2 & 4.4 & 4.3 & 4.3 \\
Metasurface II & Yes & 14.2 & 5.1 & 4.2 & 4.0 & 3.9 \\
Metasurface III & - & 13.9 & 12.0 & 10.4 & 9.9 & 9.7 \\
Metasurface IV & No & 15.6 & 10.1 & 9.8 & 9.2 & 9.1 \\
\hline
\end{tabular}

It should be reiterated that the proposed metric is independent of the choice of the evaluation plane (as in the case of bistatic performance), which seems to be much more practical having in mind that the specific spatial (and angular) relationship between the target (e.g., an aircraft) and the ground infrastructure (radar) is unknown beforehand.

\section{Conclusions}

This paper introduces a new performance metric, referred to as Normalized Partial Scattering Cross Section (NPSCS), for quantifying scattering properties of the metasurfaces. It entails evaluating the total energy scattered from the target over a solid angle. NPSCS allows for a conclusive assessment of the observability properties of the diffusion metasurface, which can be obtained in a way independent of the particulars of the setup (e.g., the choice of the evaluation plane for a bistatic mode). Moreover, the metric evaluates the average performance, which is not biased towards a particular scattering pattern. The significance and practical utility of NPSCS has been demonstrated by benchmarking two pertinent metasurface examples. The results indicate that the NPSCS complements the 
existing metrics. In particular, it can be considered a potentially attractive evaluation tool in the design of scattering metasurfaces featuring improved RCS-reduction performance. A potential disadvantage of the proposed metric is its slightly more involved formulation, which required numerical integration to evaluate NPSCS; however, when implemented using a specific programming environment, this complexity is hidden from the user.

Author Contributions: Conceptualization, M.A. and S.K.; methodology, M.A.; software, M.A. and S.K.; validation, S.S.; formal analysis, S.K.; investigation, M.A.; writing — original draft preparation, M.A.; writing - review and editing, S.K.; visualization, S.S.; supervision, S.K.; project administration, S.K. and S.S.; funding acquisition, S.K. and S.S. All authors have read and agreed to the published version of the manuscript.

Funding: This work was supported in part by the Icelandic Centre for Research (RANNIS) Grant 206606051, and by the National Science Centre of Poland Grant 2017/27/B/ST7/00563.

Conflicts of Interest: The authors declare no conflict of interest.

\section{References}

1. Sun, S.; He, Q.; Hao, J.; Xiao, S.; Zhou, L. Electromagnetic meta-surfaces: Physics and applications. Adv. Opt. Photon. 2019, 11, 380-479. [CrossRef]

2. Cui, T.J.; Smith, D.R.; Liu, R. Metamaterials: Theory, Design and Applications; Springer: New York, NY, USA, 2010.

3. Huang, F.-C.; Chiu, C.-N.; Wu, T.-L.; Chiou, Y.-P. A Circular-Ring Miniaturized-Element Metasurface With Many Good Features for Frequency Selective Shielding Applications. IEEE Trans. Electromagn. Compat. 2015, 57, 365-374. [CrossRef]

4. Li, A.; Singh, S.; Sievenpiper, D. Metasurfaces and their applications. Nanophotonics 2018, 7, 989-1011. [CrossRef]

5. Achouri, K.; Lavigne, G.; Salem, M.; Caloz, C. Metasurface Spatial Processor for Electromagnetic Remote Control. IEEE Trans. Antennas Propag. 2016, 64, 1759-1767. [CrossRef]

6. Gao, X.; Han, X.; Cao, W.-P.; Li, H.O.; Ma, H.F.; Cui, T.J. Ultrawide-band and high-efficiency linear polarization converter based on double V-shaped metasurface. IEEE Trans. Antennas Propag. 2015, 63, 3522-3530. [CrossRef]

7. Westwick, P. Stealth: The Secret Contest to Invent Invisible Aircraft; Oxford Univ. Press: London, UK, 2019; pp. 5-42.

8. Wang, Y.; Chen, K.; Li, Y.; Cao, Q. Design of nonresonant metasurfaces for broadband RCS reduction. IEEE Ant. Wireless Propag. Lett. 2021, 20, 346-350.

9. Hou, Y.; Liao, W.; Tsai, C.; Chen, S. Planar multilayer structure for broadband broad-angle RCS reduction. IEEE Trans. Antennas Propag. 2016, 64, 1859-1867. [CrossRef]

10. Kim, S.H.; Yoon, Y.J. Wideband Radar Cross-Section Reduction on Checkerboard Metasurfaces With Surface Wave Suppression. IEEE Antennas Wirel. Propag. Lett. 2019, 18, 896-900. [CrossRef]

11. Cao, T.H.X.-Y.; Gao, J.; Zhao, Y.-L.; Zhao, Y. A coding metasurface with properties of absorption and diffusion for RCS reduction. Prog. Electromagn. Res. C 2017, 75, 181-191.

12. FCosta, F.; Monorchio, A.; Manara, G. Wideband Scattering Diffusion by using Diffraction of Periodic Surfaces and Optimized Unit Cell Geometries. Sci. Rep. 2016, 6, 25458.

13. Zhao, Y.; Cao, X.; Gao, J.; Sun, Y.; Yang, H.; Liu, X.; Zhou, Y.; Han, T.; Chen, W. Broadband diffusion metasurface based on a single anisotropic element and optimized by the Simulated Annealing algorithm. Sci. Rep. 2016, 6, 23896. [CrossRef] [PubMed]

14. Yang, H.; Cao, X.; Yang, F.; Gao, J.; Xu, S.; Li, M.; Chen, X.; Zhao, Y.; Zheng, Y.; Li, S. A programmable metasurface with dynamic polarization, scattering and focusing control. Sci. Rep. 2016, 6, 35692. [CrossRef] [PubMed]

15. Chen, M.; Kim, M.; Wong, A.; Eleftheriades, G.V. Huygens' metasurfaces from microwaves to optics: A review. Nanophotonics 2018, 7, 1207-1231. [CrossRef]

16. Ji, J.; Jiang, J.; Chen, G.; Liu, F.; Ma, Y. Research on monostatic and bistatic RCS of cloaking based on coordinate transformation. Opt. 2018, 165, 117-123. [CrossRef]

17. Sui, S.; Ma, H.; Wang, J.; Pang, Y.; Feng, M.; Xu, Z.; Qu, S. Absorptive coding metasurface for further radar cross section reduction. J. Phys. D Appl. Phys. 2017, 50, 465102. [CrossRef]

18. Al-Nuaimi, M.K.T.; Hong, W.; Whittow, W.G. Aperiodic sunflower-like metasurface for diffusive scattering and RCS reduction. IEEE Ant. Wireless Propag. Lett. 2020, 19, 1048-1052. [CrossRef]

19. Ali, L.; Li, Q.; Khan, T.A.; Yi, J.; Chen, X. Wideband RCS Reduction Using Coding Diffusion Metasurface. Materials 2019, $12,2708$. [CrossRef] [PubMed]

20. Liu, X.; Gao, J.; Xu, L.; Cao, X.; Zhao, Y.; Li, S. A Coding Diffuse Metasurface for RCS Reduction. IEEE Antennas Wirel. Propag. Lett. 2017, 16, 724-727. [CrossRef]

21. Zhuanget, Y.; Wang, G.; Liang, J.; Cai, T.; Guo, W.; Zhang, Q. Flexible and polarization-controllable diffusion meta-surface with optical transparency. J. Phys. D Appl. Phys. 2017, 50, 465102. [CrossRef]

22. Rao, G.A.; Mahulikar, S.P. Integrated review of stealth technology and its role in airpower. Aeronaut. J. 2002, 106, 629-642.

23. Cui, T.J.; Qi, M.Q.; Wan, X.; Zhao, J.; Cheng, Q. Coding metamaterials, digital metamaterials and programmable metamaterials. Light. Sci. Appl. 2014, 3, e218. [CrossRef] 
24. Knott, E.F. Radar Cross Section Measurements; Springer: Berlin/Heidelberg, Germany, 2012; pp. 12-36.

25. Abdullah, M.; Koziel, S. Surrogate-Assisted Design of Checkerboard Metasurface for Broadband Radar Cross-Section Reduction. IEEE Access. 2021, 9, 46744-46754. [CrossRef]

26. Edalati, A.; Sarabandi, K. Wideband, wide angle, polarization independent RCS reduction using nonabsorptive miniaturizedelement frequency selective surfaces. IEEE Trans. Antennas Propag. 2014, 62, 747-754. [CrossRef]

27. Lee, S.-J.; Choi, I.-S.; Rothwell, E.J.; Temme, A.K. Determination of optimum bistatic angle for radar target identification. J. Electromagn. Waves Appl. 2014, 28, 551-562. [CrossRef]

28. Khan, T.A.; Li, J.; Chen, J.; Raza, M.U.; Zhang, A. Design of a Low Scattering Metasurface for Stealth Applications. Materials 2019, 12, 3031. [CrossRef] [PubMed]

29. Iriarte, J.C.; Perada, A.T.; Martinez, J.L.; Ederra, I.; Gonzalo, R.; de Maagt, P. Broadband radar cross-section reduction using AMC technology. IEEE Trans. Antennas Propag. 2013, 61, 6136-6143. [CrossRef]

30. Modi, A.Y.; Balanis, C.A.; Birtcher, C.R.; Shaman, H.N. New Class of RCS-Reduction Metasurfaces Based on Scattering Cancellation Using Array Theory. IEEE Trans. Antennas Propag. 2018, 67, 298-308. [CrossRef]

31. Koziel, S.; Abdullah, M. Machine-learning-powered EM-based framework for efficient and reliable design of low scattering metasurfaces. IEEE Trans. Microw. Theory Techn. 2021, 69, 2028-2041. [CrossRef] 